\title{
THE ROLE OF INTRAOPERATIVE NEUROPHYSIOLOGIC MONITORING TO ENHANCE SUCCESSFUL ORTHOPEDIC SURGERY
}

\author{
Panji Sananta $^{1}$, Anindita Eka Pramana Wijaya ${ }^{2}$, Marvin Anthony Putera $^{3}$
}

\author{
Correspondence: panjisananta@ub.ac.id \\ ${ }^{1}$ Orthopaedic and Traumatology Department, Faculty of Medicine, Universitas Brawijaya, Malang, Indonesia \\ ${ }^{2}$ Resident of Orthopaedic and Traumatology Department, Faculty of Medicine, Universitas Brawijaya, Malang, Indonesia \\ ${ }^{3}$ Medical Doctor at Orthopaedic and Traumatology Department, Faculty of Medicine, Universitas Brawijaya, Malang, Indonesia
}

\section{Article History: \\ Received: January 10, 2021 \\ Accepted: September 27, 2021 \\ Published: January 1, 2022 \\ Cite this as: \\ Sananta P, Wijaya AEP, Putera \\ $M A$. The role of intraoperative neurophysiologic monitoring to enhance successful orthopedic surgery. Malang Neurology Journal; 2022.8:35-38. DOI: http://dx.doi.org/10.21776/ub.mnj .2022.008.01.8}

\section{ABSTRACT}

One of the main advance in orthopaedic surgery domain has a goal to investigates the safest and harmless method in surgical procedures. Less complications means a better outcome of surgery. One of the commonest risks at orthopaedic surgery is central and peripheral nerve injury. The modality of Intraoperative Neurophysiologic Monitoring (IONM) which act to limit the risk of nerve injury during operative procedure through the evaluation of nerve integrity and function enable the surgeon to decrease injury to the nerve associated with orthopaedic surgical procedure in the operating room. This article aims to explain and describe the latest modality of IONM, its basic concept and its function at surgery. The last part of the article discussed about orthopaedic surgical techniques which use IONM. The authors hope that this article will enhance the knowledge of all the readers about IONM. This article was written based on literature study searched at Google Scholar, Medline and PubMed. The references were taken from a relatively up to date study ranging from 2013-2018. The article was selected according to the authors inclusion criteria and six articles was chosen as the references for this review. As a conclusion, IONM has an important role to increase successful rate of surgery through minimizing nerve injury risk during surgical procedure.

Keywords: Intraoperative Neurophysiologic Monitoring, nerve injury, orthopaedic surgical procedure

\section{Introduction}

Nervous system is at risk for injury during surgery with a lot of complications such as chronic pain, weakness, or even paralysis of limb which will decrease patient quality of life. The modality to monitor the function of nervous system during operative procedure is urgently needed. Intraoperative Neurophysiologic Monitoring (IONM) is integral part of surgical procedure. It was first introduced at 1935 by Foerster and Alternberger through a stimulus given directly to identify motoric cortex of patient with epilepsy. Though it was introduced at 1935, the development and commercial distribution of the machine was started at 1980s.

Nowadays IONM is often used at many kinds of surgical procedure, including orthopaedic surgery such as spine surgery, peripheral nerve surgery, dorsal rhizotomy surgery for cerebral palsy, and other surgical procedures associated with nervous system and adjacent with it. ${ }^{2}$

\section{Methods}

This review was written from studies and literatures which discuss about IONM at orthopaedic surgery. The study about this topic is still unclear and this review was composed hopefully to increase the knowledge of the readers about this topic. As the title said, this review will explain the use of IONM at increasing the successful rate of orthopaedic surgical procedure. This article also describes the basic concept of IONM and orthopaedic operative procedures which use it. Our main goal is to comprehensively review the basic concept of the usage of IONM, its function, and orthopaedic operative procedure associated with this modality through analyzing studies and literatures and review it.

Data sources was accessed to investigate previous studies. The majority of sources was from Google Scholar, Medline, and PubMed. The efficiency of investigation was done by using keyword "Intraoperative Neurophysiologic Monitoring", "role", "orthopaedic surgery". Inclusion criteria was made to ensure that the articles were standardized before analysed. The literatures must explain the introduction about basic concept of IONM, role of IONM, and the type and techniques of orthopaedic surgery which use IONM. This review was using references from studies and literatures which was published at 2013-2018. Six literatures fulfill the inclusion criteria and was used in this review. 


\section{Discussion}

\section{Basic Concept of Intraoperative Neurophysiologic Monitoring}

IONM is used to detect early changes occurring to the brain, spinal cord, or peripheral nervous system before it become permanently damaged and to identify the important nerve structure to limit nerve injury during surgery. ${ }^{1}$

\section{IONM Modality}

IONM have many modalities and each has its own specific role and it can be used together in the same time at surgery. The ideal modality comprises all of the thing mentioned below $^{3}$ :

- Uses a smooth electrode which could stick at the body surface

- Uses a wireless media

- Uses non-invasive electrode

- Uses automatic data saving and recording

- Low cost

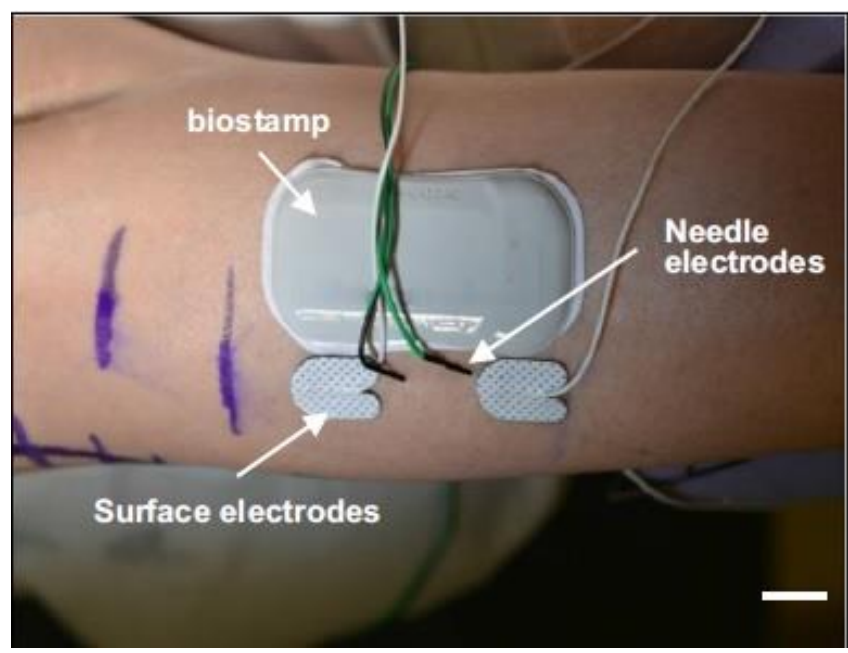

Figure 1. Example of electrode used in $\mathrm{IONM}^{3}$

\section{Modalities which often used for IONM}

\section{Motor Evoked Potentials (MEPs)}

Using Transcranial Electrical Stimulation (TES) to stimulate corticospinal tract at 5-7 series of high frequency electrical stimulus $>200 \mathrm{~Hz}$. It was chosen because TES is more resistant to anesthesia compared to Transcranial Magnetic Stimulation (TMS). ${ }^{1}$

Based on the location of its stimulation or recording, MEPs could be classified further to MEPs which recorded at muscle (Tc-mMEP) or at spinal cord (D and I wave). TcmMEP is the more popular methods because it makes stimulus and recording for MEPs easier. This method also has some flaw. It was depending to the inhaled anesthesia, the recording of Tc-mMEP needs nerve signal transmission through neuromuscular junction, the use of relaxant during surgery could affect the amplitude of Tc-mMEP, and the amplitude of Tc-mMEP has a high intertrial variability. ${ }^{1}$

Because of its limit, some studies suggest the use of D wave which obtained from TES stimulation to brain, with recording to spinal cord using epidural electrode. D wave also has some flaw. It needs an epidural electrode, a high false positive rate in scoliosis surgery, unable to observe spinal motor neuron, and a relatively low generation rate. ${ }^{1}$

\section{Somatosensory Evoked Potentials (SSEP)}

SSEP stimulation was obtained from the median nerve (MNSEP) and posterior tibial nerve (PTSEP). Though SSEP could be recorded at Erb's point, spine, or brainstem, but the recording at primary somatosensory area could give a valuable information about nerve injury during surgery. ${ }^{1}$

SSEP has long been used as IONM modality, but SSEP only evaluate sensory pathway through the dorsal column of spinal cord and it cause a risk of undetected injury to motoric pathway. The advantage of SSEP is it does not cause unnecessary move during surgery, easy to calculate, and has a relatively low intertrial variability compared to MEPS. SSEP is a specific but less sensitive modality to detect ongoing nerve injury. ${ }^{1}$

\section{Electroencephalography (EEG)}

EEG record electrical activity from scalp. EEG was first introduced as IONM at 1960s for carotid endarterectomy surgery and still used up to these days to evaluate cerebral perfusion during vascular surgery, to observe the depth of anesthesia, and hypothermia degree. The sensitivity of EEG is still lower than Transcranial Dopller. ${ }^{1}$ EEG has an essential capability to detect a rather perplexing clinical condition such as non-convulsive status epilepticus which is a neurological emergency. Clinical manifestation of nonconvulsive status epilepticus could be subtle and easily missed in the form of confusion and anxiety but the change and disruption in EEG wave shows an epileptic state. ${ }^{4}$ Other type of EEG is video assisted EEG which is frequently utilized as an integral part in differentiating seizure and psychogenic non-epileptic seizures. ${ }^{5}$

\section{Electromyography (EMG)}

EMG records electrical activity produced by muscles. Recording technique of EMG include free running EMG, stimulated EMG, and intraoperative nerve conduction studies. Free running EMG detects mechanic and metabolic irritation of the nerve and could record muscles innervated by those nerve without electrical stimulation from the nerve. Stimulated EMG was done to give electrical stimulation to the nerve. ${ }^{1}$

EMG usage during surgery was limited to detect nerve injury because it could only detect it if there is direct irritation to the nerve, such as because stretching or heat from electrocautery. EMG does not give any information about functional integrity of the nerve and could not detect any microvascular problem at the nerve. ${ }^{1}$

\section{Brainstem Auditory Evoked Potential (BAEPs)}

BAEPs record a small electrical potential which emerge because of acoustic stimulus. This potential action relatively resistant to anesthesia so it could be used to evaluate auditory structure during surgery. ${ }^{1}$

\section{Visual Evoked Potential (VEP)}

VEP is an electrical potential which sparked from visual stimulation which recorded from scalp above the visual cortex. IONM visual tract is needed at some surgical procedure such as transsphenoid surgery, aneurysm clipping, and tumor surgery which located at or near at visual nerve. ${ }^{1}$ 


\section{IONM Application at Orthopaedic Surgery}

\section{Spine Surgery}

At 2012, the American Academy of Neurology (AAN) and American Clinical Neurophysiology Society (ACNS) recommended the combination of SSEPs, EMG, and TcMEP at spine surgery to predict adverse event such as paraparesis, paraplegia, and quadriplegia. With the addition of EMG, additional information about the procedure location of the nerve root, traction or irritation of the nerve root could also be obtained. The accuracy of pedicle screw insertion could also be decided with calculation of electrical conduction between pedicle screw and the nerve root using stimulated EMG. ${ }^{1}$

\section{Peripheral Nerve Surgery}

Tumor surgery at or near at peripheral nerve or nerve sheath could induce risk of nerve lesion. Peripheral nerve IONM could be done by measuring nerve potential action with the use of MEP, SSEP, and DES. DES could map nerve fiber in the area of the tumor and could identify motoric axon and monitor motor nerve system. ${ }^{1}$

\section{Pelvic and Acetabulum Surgery}

One of the most feared complication of pelvic and acetabulum surgery is iatrogenic nerve injury to sciatic and femoral nerve. The incident is varied from 1 until $18 \%$ with $24 \%$ of it become permanently debilitated. Injury usually triggered by the use of retractor or plate placement pinching the nerve or the vascular supplying those nerve. The use of IONM could decrease the incident of nerve injury by early detection so the surgeon could modify the procedure such as change in approach, retractor replacement, or increasing the mean arterial pressure (MAP). At 2013 Porat et al states that the use of EMG only could not detect nerve injury accurately and they recommend Tc-mMEP and PNSEP (peroneal nerve somatosensory evoked potentials). ${ }^{6}$

\section{Selective Dorsal Rhizotomy for Cerebral Palsy}

Child with cerebral palsy which has a spastic diplegia is severely debilitated and the condition could go worse. When the spasticity become resistant to physiotherapy, other treatment such as botulinum injection and surgery will become options to restore the posture and gait through reduction of overt muscle tone. ${ }^{7}$

Surgical procedure which could be done is selective dorsal rhizotomy (SDR). First introduced by Foerster at 1898, until these days, this procedure still spark a lot of controversies because of its irreversible effect. The challenge was to obtain effective and selective result. The target of SDR is to cut partially the dorsal root of lumbar spine II until sacral I or II with the aim to decrease spasticity and increase the range of motion to preserve muscle power. ${ }^{7}$

If the nerve fiber cut was too much, it will weaken the muscle tone needed while cut too little will produce undertreatment. To achieve the targeted root and muscle group, it needed the IONM modality. ${ }^{8}$

Some IONM modality was developed in recent years with principle ${ }^{8}$ :

- Ventral root stimulation to decide the root level and was done orthodromically. With $2 \mathrm{~Hz}$ frequency and low intensity $(200 \mathrm{mcA})$, stimulation was done starting at the level of second lumbar spine which mediate hip flexion. Stimulation was continued to the level of sacral II/III to observe anal sphincter response.

- Dorsal root stimulation was used to evaluate the degree of excitability from metameric circuitries. Stimulation was done globally using $50 \mathrm{~Hz}$ frequency with $0,1 \mathrm{msec}$ suration. This intensity is 3-5 times above the motoric limit, so that it may not trigger too many "parasite" diffusion

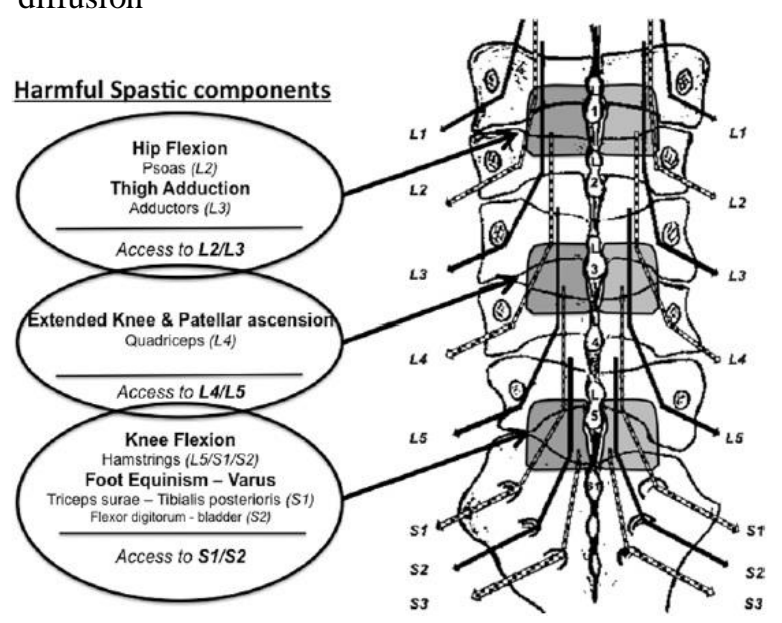

Figure 2. Spastic component at Cerebral Palsy ${ }^{5}$

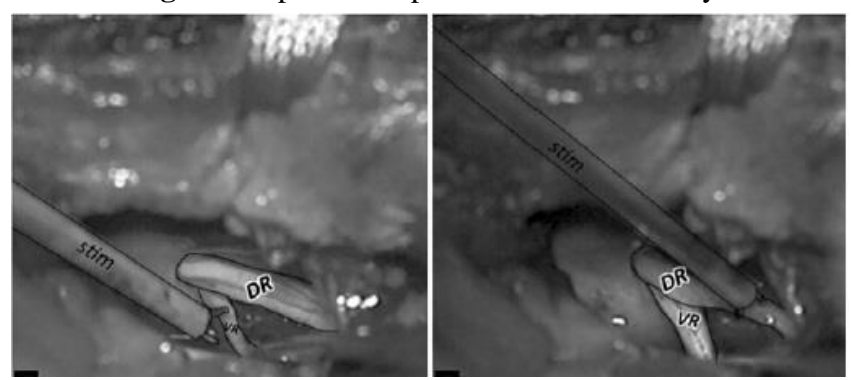

Figure 3. Ventral root and Dorsal root stimulations ${ }^{6}$

Root stimulation was done using bipolar probe with interpole distance of $4 \pm 1 \mathrm{~mm}$. Stimulation was done for a few seconds and was done for three times in a row, and the associated muscle response was recorded. ${ }^{8}$ Muscle response to stimulation was interpreted using criteria described by Fasano, and divided to grade $0,1,2,3$, and 4 showed in Table 1.

Tabel 1. Interpretation of muscle response grading to stimulation by Fasano

\begin{tabular}{cl}
\hline Grade & Description \\
\hline 0 & $\begin{array}{l}\text { Muscle response happens at muscle group at the level } \\
\text { of dorsal root stimulated with single motor response } \\
\text { (normal) }\end{array}$ \\
\hline 1 & $\begin{array}{l}\text { There is increasing amplitude at the stimulated } \\
\text { muscle group }\end{array}$ \\
\hline 2 & $\begin{array}{l}\text { There is increasing amplitude at adjacent muscle with } \\
\text { the muscle group which stimulated }\end{array}$ \\
\hline 3 & $\begin{array}{l}\text { There is increasing amplitude at the muscle group } \\
\text { distant from the muscle group which stimulated }\end{array}$ \\
\hline 4 & $\begin{array}{l}\text { Diffuse muscle response at the muscle group at } \\
\text { contralateral extremity and upper extremity }\end{array}$ \\
\hline
\end{tabular}

\section{Scoliosis Reconstruction}

Scoliosis reconstruction commonly produce a good outcome for patient comfort and postural alignment but this procedure also carries a great post-operative complication, especially the one with neuromuscular type scoliosis. The 
most feared complication is paralysis. IONM with SSEPs and TceMEPs has been used for a long time as a modality to monitor neurological status during scoliosis reconstruction surgery. Unfortunately, this IONM modality could not detect the possible spinal cord injury. ${ }^{1}$

\section{Conclussion}

Intraoperative Neurophysiologic Monitoring has an important role at orthopaedic surgery to minimize nerve injury risk during surgical procedure so it could increase the success rate of the surgery.

\section{Conflict of Interest}

The authors declare that there is no conflict of interest

\section{Acknowledgement}

I am grateful to all of those with whom I have had the pleasure to work during this paper. Each of colleagues in Orthopaedic Department have provided me extensive personal and professional guidance and taught me a great deal about both scientific research and life in general.

\section{References}

1. Kim, Sung-Min., Kim, Seung Hyun., Seo, Dae-Won., and Lee, Kwang-Woo. Intraoperative Neurophysiologic Monitoring: Basic Principles and Recent Update. Journal of Korean Medical Sciences 2013; 28: 1261 1269. DOI: $10.3346 / \mathrm{jkms} .2013 .28 .9 .1261$

2. Georgoulis, G., Brinzeu, A., and Sindou, M. Dorsal Rhizotomy for Children with Spastic Diplegia of
Cerebral Palsy Origin: Usefulness of Intraoperative Monitoring. Journal of Neurourgery Pediatric. 2018; 22: 89 - 101. DOI: 10.3171/2018.1.PEDS17577

3. Liu, Y., Tian, L., Raj, M. S., Cotton, M., Ma, Y., Ma, S., et al. Intraoperative Monitoring of Neuromuscular Function with Soft, Skin - Mounted Wireless Devices. Nature Partner Journals. 2018; 1: 19. DOI: $10.1038 / \mathrm{s} 41746-018-0023-7$

4. Pramesti FA, Husna M, Kurniawan SN, Rahayu M. Diagnosis and Management of Nonconvulsive Status Epilepticus. Malang Neurology Journal. 2017; 3(1): 3038. DOI: 10.21776/ub.mnj.2017.003.01.6

5. Parameswaran S, Vijayamma AKT, Pillai AM. Efficacy of Short Term Video EEG in Detecting Psychogenic Non-Epileptic Seizures. Malang Neurology Journal. 2019; 5(2): 65-67. DOI: 10.21776/ub.mnj.2019.005.02.3

6. Porat, M., Orozco, F., Goyal, N., Post, Z., and Ong, A. Neurophysiologic Monitoring Can Predict Iatrogenic Injury During Acetabular and Pelvic Fracture Fixation. Hospital for Special Surgery Journal. 2013; 9: 218 222. DOI: $10.1007 / \mathrm{s} 11420-013-9347-7$

7. Hammett, T.C., Boreham, B., Quraishi, N. A., and Mehdian, S. M. Intraoperative Spinal Cord Monitoring During the Surgical Correction of Scoliosis due to Cerebral Palsy and Other Neuromuscular Disorders. European Spine Journal. 2013. 22 (Suppl 1): 538 - 541. DOI: $10.1007 / \mathrm{s} 00586-012-2652-\mathrm{x}$

8. Salehi, S., Nemati, H., and Soltani, A. Intraoperative Neurophysiology Monitoring During Selective Dorsal Rhizotomy for Spastic Cerebral Palsy. Iranian Journal of Neurosurgery. 2018; 4: 117 - 122.

DOI: 10.32598/irjns.4.2.117 